\title{
Spectrum of Meningitis in Adult at the Douala General Hospital
}

\author{
Yacouba N. Mapoure1,2, Henry N. Luma',3, Dieudonné Gnonlonfoun4, Paul M. Ossou-Guiet5, \\ Xaverie Biloa1, Jacques N. Doumbe1,2, Hugo Bertrand N. Mbatchou1,2, \\ Benjamin Clet N. Tchaleu6, Julius Y. Fonsah7, Albert S. Mouelle ${ }^{1,2}$, Alfred K. Njamnshi, \\ ${ }^{1}$ Department of Clinical Sciences, The University of Douala, Douala, Cameroon \\ ${ }^{2}$ Department of Clinical Sciences, Douala General Hospital, Douala, Cameroon \\ ${ }^{3}$ Department of Internal Medicine, The University of Yaoundé I, Yaoundé, Cameroon \\ ${ }^{4}$ Department of Neurology, National University Hospital Centre, Cotonou, Bénin \\ ${ }^{5}$ Department of Neurology, Brazzaville University Teaching Hospital, Brazzaville, Congo \\ ${ }^{6}$ Department of Clinical Sciences, Université des Montagnes, Douala, Cameroon \\ ${ }^{7}$ Department of Neurology, Yaoundé Central Hospital, Yaoundé, Cameroon \\ ${ }^{8}$ Department of Neurology, The University of Yaoundé I, Yaoundé, Cameroon \\ Email: mapourevacouba@gmail.com
}

Received 1 January 2014; revised 1 February 2014; accepted 8 February 2014

Copyright (C) 2014 by authors and Scientific Research Publishing Inc.

This work is licensed under the Creative Commons Attribution International License (CC BY).

http://creativecommons.org/licenses/by/4.0/

c) (i) Open Access

\section{Abstract}

Objectives: Describe the clinical, aetiological and prognostic features of infectious meningitis in adults at the Douala General Hospital (DGH). Patients and Methods: We carried out a 5-year retrospective cross-sectional study at the DGH, on data from the registers of the bacteriology and biochemistry laboratories. Cases of meningitis were identified from the results of the cerebrospinal fluid (CSF) analysis, and the patient clinical file was obtained. Cases files of patients aged 15 years and above were included. For each patient, sociodemographic, clinical features and hospital mortality data were extracted. Results: During study period, 1877 CSF analyses were recorded and 135 were enrolled for data analysis. Up to 74 patients $(55 \%)$ were male and the mean age was $40.04 \pm 12.5$ years. The time lapse between the onset of symptoms and consultation was $12.24 \pm$ 11.16 days. The main clinical signs were meningeal syndrome $(96.3 \%)$, neck stiffness $(71.1 \%)$, reduced alertness $(64.4 \%)$ and confusional states $(55.6 \%)$. The main aetiology was bacterial (45.19\%) with Streptococcus pneumoniae $(45.90 \%)$ and Neisseria meningitis $(29.51 \%)$ leading. The other aetiologies were viral (21.48\%), fungal with Cryptococcus neoformans (20\%) and meningeal tuberculosis (13.33\%). Fifty eight patients $(42.9 \%)$ had a positive HIV serology. The mean duration of hospitalization was $9.05 \pm 8.1$ days, and the total in-hospital mortality was $25.18 \%$. 117 patients $(86.8 \%)$ had received antibiotherapy before diagnosis. Conclusion: The clinical features of meningitis in the DGH are as in classical description. However, the mortality is high and 
the major aetiologies appear to be associated with HIV infection at least in part. Prospective multi-centric studies are needed to provide more evidence for the development of staged management guidelines in our resource-limited settings.

\section{Keywords}

Meningitis, Signs, Aetiologies, Mortality, Adults, Douala, Cameroon

\section{Introduction}

In spite of the progress made in diagnosing and treating of meningitis, it is still a problem in sub-Saharan Africa due to its high frequency and serious complications. Since the advent of HIV infection, there has been a modification of the picture of meningitis with the significant emergence of Cryptococcus neoformans in adults. In Cameroon, few studies exist on adult meningitis, although epidemics have been reported in the northern part of the country [1] [2]. Data on the aetiologies of meningitis apart from epidemics periods are limited [3] [4]. In order to contribute to the better management of meningitis, we decided to study the clinical spectrum and aetiologies of infectious meningitis in adults at the Douala General Hospital (DGH).

\section{Patients and Methods}

It was a retrospective cross-sectional study carried out at the DGH over a period of 5 years from January 1, 2008 to December 31, 2012. Using the registers of the bacteriology and mycology laboratory, we identified records of all cerebrospinal fluid (CSF) analyzed during the study period. All CSF with characteristics of meningitis defined by the presence of an identified germ and/or the presence of at least 10 leucocytes per ml of CSF were selected. We then included into the study cases file of patients aged 15 years and above. From the list of patients obtained in the laboratory, we identified the ward/service where the patient was hospitalized notably the internal medicine or reanimation service in order to retrieve the case file. Case file of all patients treated empirically for meningitis were not included in the study. For each case file, data was collected in a unique data entry form for all patients. Data collected included sociodemographic information (sex, age, place of residence, occupation, matrimonial status), medical history, time taken to treat, clinical and CT scan information, as well as the clinical outcome from which mortality was determined.

\subsection{Definition of Terms}

We considered as viral meningitis all CSF results with the following characteristics: pleiocytosis with lymphocyte predominance, normal glucose in CSF, normal or increased protein level in CSF, and the absence of a germ on direct examination and culture on specific media.

The diagnosis of cryptococcal meningitis was based on the presence of Cryptococci on India ink staining or on culture on Sabouraud medium.

Tuberculosis meningitis was diagnosed on the basis of the CSF characteristic: notably lymphocyte-predominant pleiocytosis, low glucose level, high protein level, low chloride level and the absence of any germ on direct examination or culture.

We considered as Listeria monocytogene meningitis, CSF with mixed pleiocytosis, low glucose, high protein level, and Gram positive cocci on bacteriologic examination.

Apart from bacteriology examination, the diagnosis of bacterial meningitis was based on cloudy aspect and/or neutrophil-predominant pleiocytosis of the CSF, low glucose and high protein levels, and the presence of germs on direct microscopy or culture.

\subsection{Statistical Analysis}

Data were analysed using SPSS software version 16.0 for Windows. Quantitative variables were expressed as means plus or minus standard deviation while qualitative variables were expressed as percentages. 


\subsection{Ethical Considerations}

Ethical clearance was obtained from the ethical committee of the Faculty of Medicine and Pharmaceutical Sciences of the University of Douala as well as that of the DGH. Data collection and handling was confidential and the identity of the patients was well preserved.

\section{Results}

During the study period, 1877 CSF had been analyzed (Figure 1) and 135 case files were included. The mean age of the patients was $40.04 \pm 12.5$ years with interval from 16 to 65 years. A hundred and thirty patients (83.37\%) were enrolled in the internal medicine and 22 (16.63\%) in the intensive care unit. Table 1 and Table 2 show the other sociodemographic characteristics and past medical history respectively. Among the HIV-positive patients, 29 (21.48\%) were on antiretroviral treatment (ARV) of whom 27 had first line and 2 on second line treatment. HIV infection was found in patients with cryptococcal meningitis (27 cases), tuberculosis meningitis (18 cases), bacterial meningitis (7 cases) and viral meningitis (6 cases).

The mean duration between onset of symptoms and initial consultation was $12.24 \pm 11.16$ days (range: 1 to 60 days) while the duration between onset of symptoms and the diagnosis was $14.41 \pm 11.56$ days (range: 1 to 71 ).

Table 3 shows the clinical parameters at admission. Fever was present in 108 patients (80\%) while 22 (16.30\%) patients had a Glasgow Coma Scale (GCS) of $<9 / 15$ of whom 4 had septic shock. The neurological signs (Table 4) were dominated mainly by the meningeal syndrome in 130 cases (96.3\%), neck stiffness in 96 cases (71.1\%) followed by an altered level of consciousness in 87 cases (64.4\%). Cranial nerve involvement was observed in 14 patients (10.4\%). This cranial nerve involvement concerned one nerve in 7 patients, two nerves in three patients, and more than two nerves in four patients. The affected cranial nerves were mainly the facial (5 cases), the sixth (4 cases), the third (3 cases) and the eighth (2 cases). Epileptic seizures were generalised in four patients, focal in three, and focal with secondary generalization in one case, and there was status epilepticus in two cases. The other complications were mainly hypoacousia in seven patients, five of whom had pneumococcal meningitis and two had tuberculosis meningitis.

Up to one hundred and sixteen patients (85.93\%) had had a cerebral CT scan before the lumbar tap and among them, 23 with contrast-enhancement. The main CT scan abnormalities were cerebral atrophy (17 cases), ventricular dilatation (11 cases), cerebral oedema (7 cases) and cerebral ischaemia (2 cases). Nine out of 23 patients (39.13\%) had meningeal enhancement after contrast medium injection. One hundred and eight patients

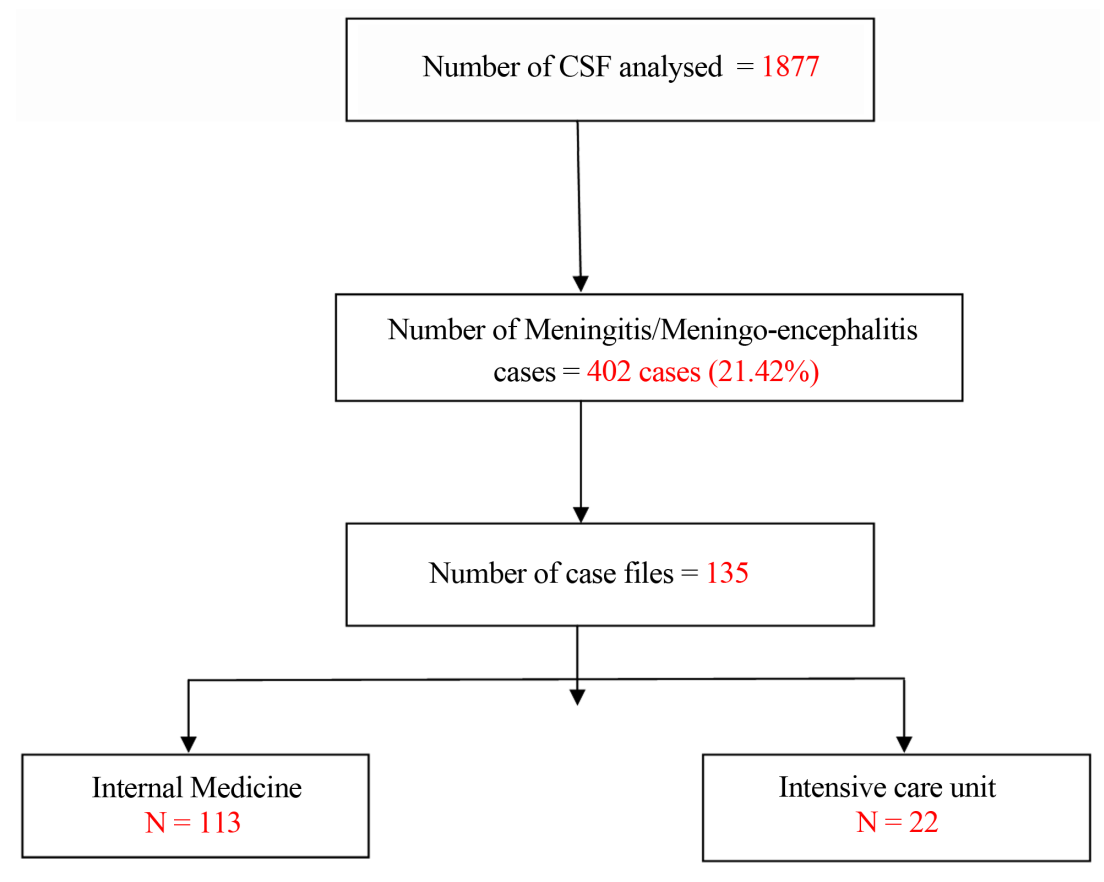

Figure 1. Flowchart of patients followed up for meningitis. 
Table 1. Sociodemographic characteristic of patients.

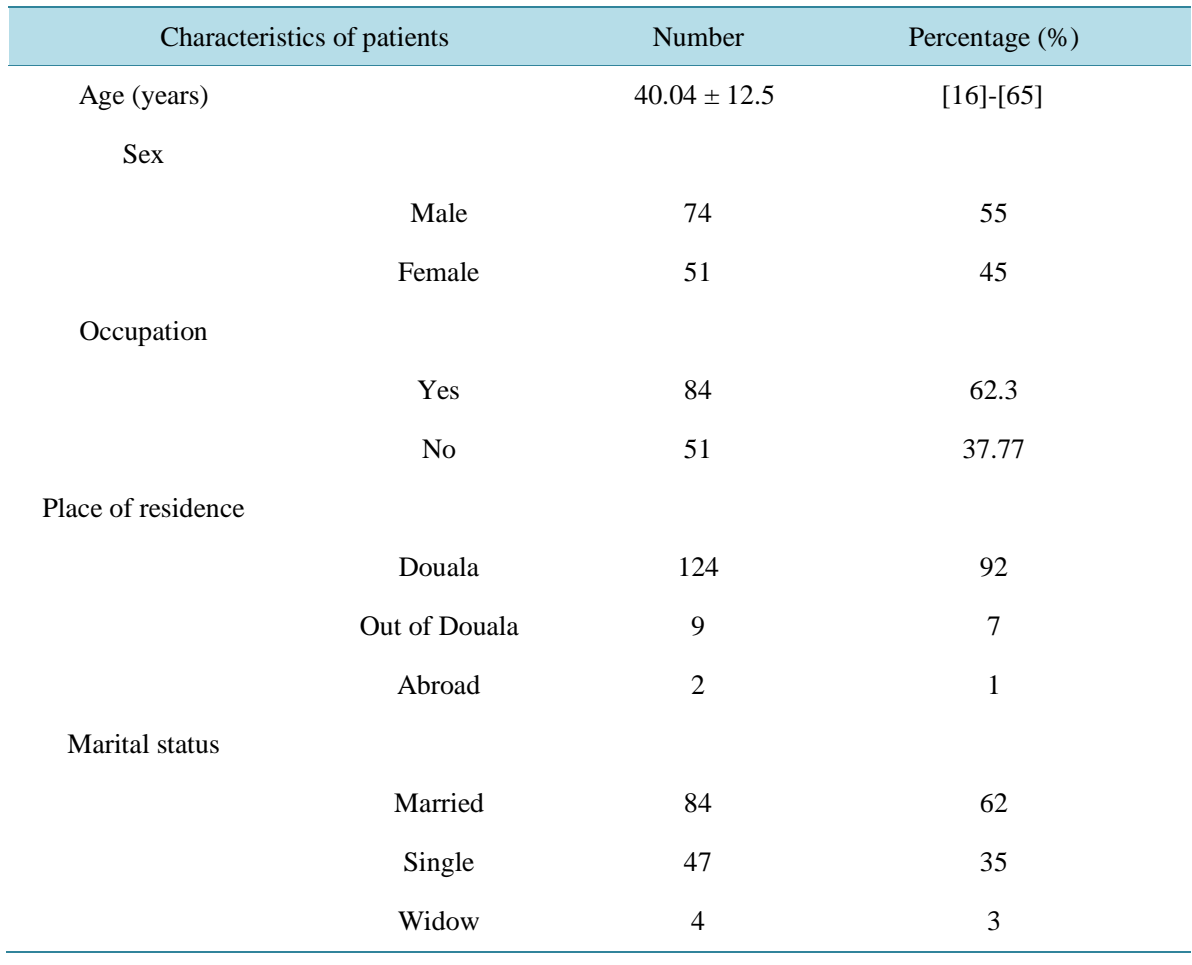

Table 2. The main past medical history of the patients.

\begin{tabular}{llcc}
\hline \multicolumn{1}{c}{ Past medical history } & & Number & Percentage (\%) \\
\hline HIV infection & Antiretroviral treatment & 58 & 42.96 \\
& WHO AIDS Classification & 29 & 50 \\
& Stage 1 & 2 & 3.45 \\
& Stage 2 & 2 & 17.24 \\
& Stage 3 & 10 & 12.07 \\
& Stage 4 & 7 & 67.24 \\
Chronic alcoholism & & 39 & 14.81 \\
Pulmonary tuberculosis & & 20 & 11.85 \\
Arterial hypertension & & 16 & 5.18 \\
Meningitis (of unknown cause) & 7 & 3.7 \\
Cancer & & 5 & 2.22 \\
Diabetes mellitus & 3 & 2.22 \\
Sickle cell disease & 3 & 1.48 \\
Cerebral toxoplasmosis & & 2 & 1.48 \\
Herpes Zoster & & 2 & 0.74 \\
Kaposi sarcoma & & 3 &
\end{tabular}


Table 3. Patients vital signs at admission.

\begin{tabular}{ccccc}
\hline Sign & Number & Mean \pm SD & Minimum & Maximum \\
\hline Temperature $\left({ }^{\circ} \mathrm{C}\right)$ & 135 & $38.44 \pm 1.02$ & 37 & 40 \\
SBP $(\mathrm{mm} \mathrm{hg})$ & 135 & $124.75 \pm 34.07$ & 54 & 230 \\
DBP (mm hg) & 135 & $79.66 \pm 13.74$ & 40 & 120 \\
Pulse (pulsations/min) & 135 & $108.74 \pm 24.54$ & 48 & 170 \\
Glasgow Coma Scale & 135 & $12.27 \pm 2.97$ & 3 & 15 \\
\hline
\end{tabular}

SBP: Systolic Blood Pressure; DBP: Diastolic Blood Pressure.

Table 4. Neurologic signs and symptoms during meningitis in adult.

\begin{tabular}{ccc}
\hline Signs and symptoms & Frequency & Percentage (\%) \\
\hline Triad of meningeal signs ${ }^{*}$ & 130 & 96.3 \\
Neck stiffness & 96 & 71.1 \\
Altered level of consciousness & 87 & 64.4 \\
Confusional states & 75 & 55.6 \\
Cerebellar signs & 32 & 23.7 \\
Motor deficit of the limbs & 18 & 13.3 \\
Sensitive deficit of the limbs & 16 & 11.9 \\
Cranial nerve lesions & 14 & 10.4 \\
Epileptic seizures & 10 & 7.4 \\
\hline
\end{tabular}

Triad of meningeal signs: headache, vomiting, constipation.

Table 5. Aetiologies and mortality of meningitis in the adult.

\begin{tabular}{|c|c|c|c|c|c|c|}
\hline \multirow[t]{2}{*}{ Pathogenic agent } & \multicolumn{3}{|c|}{ Number of cases } & \multicolumn{3}{|c|}{ Mortality } \\
\hline & Number & $\% \dagger$ & $\% \S$ & $\mathrm{N}$ & $\% †$ & $\% \S$ \\
\hline Bacteria & 61 & - & 45.19 & 12 & 19.67 & 8.89 \\
\hline Streptoccocus sp. & 28 & 45.90 & 20.74 & 4 & 9.83 & 2.96 \\
\hline Neisseria sp. & 18 & 29.51 & 13.33 & 4 & 9.93 & 2.96 \\
\hline S. agalatiae & 4 & 6.56 & 2.96 & 2 & 3.28 & 1.48 \\
\hline Haemophilus sp. & 3 & 4.92 & 2.22 & 2 & 3.28 & 1.48 \\
\hline Listeria sp. & 2 & 3 & 1.48 & 0 & 0 & 0 \\
\hline Others & 6 & 9.83 & 4.44 & 2 & 3.28 & 1.48 \\
\hline Viral & 29 & - & 21.48 & 5 & 17.24 & 3.70 \\
\hline Cryptococcus sp. & 27 & - & 20 & 11 & 40.74 & 8.15 \\
\hline Tuberculosis & 18 & - & 13.33 & 6 & 33.33 & 4.44 \\
\hline Total & 135 & - & 100 & 34 & 100 & 25.18 \\
\hline
\end{tabular}

$\% \dagger$ : Percentage with respect to the subgroup of the cause; \%§: Percentage with respect to the global frequency. 
(80\%) had clear lymphocytic CSF, 26 patients (19.26) had cloudy neutrophil-predominant CSF and in one patient the CSF had a variably mixed cellular constitution. Forty-eight percent of the patients had a clinical presentation of meningitis and $52 \%$ who had a presentation of meningo-encephalitis.

The main causes of meningitis were bacterial infections, the Streptococcus pneumoniae being the principal pathogenic agent (Table 5). One hundred and seventeen (86.8\%) had received prior antibiotic before the diagnosis of meningitis. Virological tests were not been done in the CSF in this study. The mean duration of hospitalization was $9.05 \pm 8.1$ days and the hospital mortality was $25.18 \%$. In the intensive care unit, 10 patients had died (45.45\%) against 22 patients in internal medicine (19.47\%). The fungus Cryptococcus sp. appeared as the main pathogenic agent with the highest case fatality rate (40.74\%).

\section{Discussion}

This study has allowed us to appreciate the clinical and aetiological spectrum of meningitis in a non-epidemic context in a university teaching hospital in Douala. The mean age of our patients was 40.04 years similar to that reported by other authors [3] [5] [6]. Of 58 (46.96\%) HIV-infected patients, 79.31\% were classified in the WHO stage 3 - 4 of AIDS. Two South African studies on the aetiologies of adult meningitis estimated HIV prevalence at $37.3 \%$ [7] and $66.7 \%$ [8] while a Central African study carried out solely on bacterial meningitis of the adult estimated it at 55.1\% [6]. Other studies do not mention this prevalence [3] [5] [9].

The principal clinical aspects revealed in this study were the meningeal syndrome (96.3\%), neck stiffness (71.1\%), altered level of consciousness (64.4\%) and a confusional syndrome (55.6\%). These findings can orientate the general practioner who is often in a remote and isolated area in our context without adequate diagnostic facilities that would contribute to optimal treatment. Varlet et al. [10] tried to determine the sensitivity and specificity of signs in meningitis and found that headache, vomiting or fever had a sensitivity lower than 30\% against $40 \%$ for neck stiffness. They also demonstrated that the absence of two signs notably fever, headaches, neck stiffness and altered level of consciousness had a negative predictive value of 95\%. However, one has to take into account the effect of cryptococcal meningitis for which $74 \%$ of cases, the meningeal syndrome is incomplete, often reduced to incoherent speech, and behavioral disturbances [11]. Other authors [5] reported as main signs neuropsychological disturbances (100\%), coma (29\%) and hemiplegia (6\%). These signs which translate brain involvement may be explained by the long delays before consultations. The complications and sequellae were insufficiently assessed in our study because of incomplete files with the retrospective nature of the study.

Bacterial infections (45.18\%) represented the first cause of meningitis and Streptococcus pneumoniae (45.9\%) and Neisseria meningitidis (29.51\%) were the main agents. If the main bacterial aetiologies in this study are similar to those of Koulla et al. [3] in 1997 in Cameroon, the proportions are not the same. In the latter study, they were lower with respectively $30.4 \%$ and $13 \%$ for Streptococcus pneumoniae and Neisseria meningitidis. This difference could be explained by their relatively small sample of 70 patients studied; of which 40 had a bacteriological diagnosis due to the inability of the patients to pay for this test. Pneumococcus is reported as the most frequent causal agent of adult meningitis with proportions varying from $45.2 \%$ to $77.7 \%$ [5]-[7] [9] [12]. However, two african studies have reported the Pneumococcus as the second most frequent aetiologic agent of bacterial meningitis with proportion of $37.1 \%$ in a Tunisian study coming after Haemophilus influenzae [13] and 4.8\% coming after Meningococcus in Mozambique [14]. This low proportion in the Mozambican study may be explained by the laboratory difficulties encountered in doing precise bacteriologic diagnosis. Besides, a South African study [7] carried out on 284 patients with meningitis of whom $37.3 \%$ were HIV positive, found as main aetiologies: tuberculosis meningitis (25.4\%), bacterial causes (22.5\%), viral causes (14.1\%) and Cryptococcus sp (13\%). Another South African study [8] carried out on 60 cases of meningitis with 66.7\% HIV infections still confirms the predominance of tuberculosis (16.67\%) and other bacteria (16.67\%), followed by aseptic meningitis (15\%), cryptococcal meningitis (15\%), and neurosyphilis (3.33\%). These results suggest differences in aetiology as a function of geographical location. The proportion of HIV infection alone may not explain this difference since our study also has a high HIV prevalence but with a lower number of tuberculosis meningitis. Other factors such as environmental factors may also influence the occurence of meningitis. All cases of cryptococcal meningitis in our study were diagnosed in HIV-infected patients. It should be noted that our study could not identify the specific aetiologies in the viral meningitis group as the necessary laboratory tests are not yet available in our hospital. Furthermore, a few of these cases could represent HIV-associated aseptic meningitis but this could not be proven. Also, for bacteriologic studies, antibiotic sensitivity tests are not performed. 
The general in-hospital mortality was $25.18 \%$ and this was higher in cryptococcal meningitis cases (8.15\%). The mortality from bacterial meningitis was $8.89 \%$ largely lower than $27.1 \%$ reported by Koulla et al. in 1997 [3]. In Gabon [5], the mortality during acute bacterial meningitis was $18 \%$ and it was $31.9 \%$ in the Central African Republic [6]. The factors associated with the risk of death are age greater than 50 years [3], HIV positivity, low CD4 level [7], delay in arrival at the hospital and immunodepression from HIV or malnutrition [8]. The duration of hospitalization in the current study was 9.05 days as against 14.02 days in the Central African Republic [6]. In our study, Cryptococcus neoformans was the aetiologic agent with the highest number of deaths (40.74\%). This result was similar to in-hospital mortality of $42.2 \%$ reported by Mbuagbaw et al. within 21 days of hospitalization [3]. The global mean duration of hospitalization was $9.05 \pm 8.1$ days. In studying bacterial meningitis, Tsiba et al. [6] in the Central African Republic reported a mean duration of hospitalization of 14.2 days. This difference with the current study arises from the fact that our study included viral meningitis whose duration of hospital stay is often shorter.

If the weakness inherent to most retrospective studies coupled with the absence of some laboratory analysis, it has the strengths providing some epidemiological, clinical and biological evidence of the development of staged management guidelines that will meet the challenges of our pyramidal system. Future multi-centre prospective studies should aim at filling these knowledge gaps.

\section{Conclusion}

The clinical features of meningitis in the DGH are as in classical description. However, the mortality is high especially with cryptococcal meningitis and the major aetiologies appear to be associated with HIV-infection at least in part. Bacterial agents are the most frequent cause. Prospective multi-centre studies are needed to provide more evidence for the development of staged management guidelines.

\section{References}

[1] Massenet, D., Inrombe, J. and Mevoula, D.-E. (2009) Serogroup W135 Meningitis Northern 2007-2008. Emerging Infectious Diseases, 15, 340-342. http://dx.doi.org/10.3201/eid1502.080988

[2] Massenet, D., Vohod, D., Hamadicko, H. and Caugant, D.A. (2011) Epidemic Meningococcal Meningitis, Cameroon. Emerging Infectious Diseases, 17, 2070-2072. http://dx.doi.org/10.3201/eid1711.110468

[3] Koulla, S., Mbuagbaw, J., Kuaban, C. and Kouda Zeh, A. (1997) Etiological and Outcome of Adult Bacterial Meningitis in Yaoundé, Cameroon. International Journal of Infectious Diseases, 2, 9-11. http://dx.doi.org/10.1016/S1201-9712(97)90003-5

[4] Mbuagow, J.N., Biholong and Njamnshi, A.K. (2006) Cryptococcal Meningitis and HIV in the Internal Medicine Service at the Yaoundé University Hospital, Cameroon. AJNS, 25, 13-18.

[5] Okome-Nkoumou, M. and Loembe, P.M. (1999) Les méningites bactériennes de l'adulte. Étude de 85 cas observés dans l'unité des maladies infectieuses de la Fondation Jeanne Ebori, Libreville, Gabon. Bulletin de la Société de pathologie exotique, 92, 288-291.

[6] Tsiba, J.B. and Nzingoula, S. (2006) Les méningites bactériennes de l'adulte en milieu hospitalier centrafricain. Bulletin de la Société de pathologie exotique, 99, 261-263.

[7] Bergemann, A. and Karstaedt, A.S. (1996) The Spectrum of Meningitis in a Population with High Prevalence of HIV Disease. QJM, 89, 499-504. http://dx.doi.org/10.1093/qjmed/89.7.499

[8] Silber, E., Sonnenberg, P., Ho, K.C., Koornhof, H.J., Eintracht, S., Morris, L. and Saffer, D. (1999) Meningitis in a Community with a High Prevalence of Tuberculosis and HIV Infection. Journal of the Neurological Sciences, 162, 2026. http://dx.doi.org/10.1016/S0022-510X(98)00259-7

[9] Dao, S., Goita, D., Oumar, A.A., Diarra, S., Traore, S. and Bougoudodgo, F. (2008) Aspects épidémiologiques des méningites purulentes au Mali. Médecine d'Afrique noire, 55, 514-518.

[10] Varlet, G., Bazeze, V. and N’Dri, O.K.A. (2009) Sensibilité et spécificité des signes cliniques chez l'adulte-Côte D’Ivoire. Médecine et Maladies Infectieuses, 39, 445-451. http://dx.doi.org/10.1016/j.medmal.2009.06.015

[11] Njamnshi, A.K., de P. Djientcheu, V., Bissek, A.C., Ongolo-Zogo, P., Mapoure, N.Y., Yepnjio, F.N., Sini, V., Dongmo, L., Kaptue, L.N. and Muna, W.F.T. (2003) Epidemiological and Aetiological Features of Nervous System HIVAIDS in Yaoundé: A Preliminary Study. Health Services Research Journal, 3, 18-28.

[12] Owusu, M., Nguah, S.B., Boaitey, Y.A. and Adu-Boateng, E.B. (2012) Aetiological Agents of Cerebrospinal Meningitis: A Retrospective Study from a Teaching Hospital in Ghana. Annals of Clinical Microbiology and Antimicrobials, 11, 
28. http://dx.doi.org/10.1186/1476-0711-11-28

[13] Mezghani Maalej, S., Kassis, M., Mahjoubi Rhimi, F., Damak, J. and Hammami, A. (2006) Bactériologie des méningites communautaires dans la région de Sfax, Tunisie. Médecine et Maladies Infectieuses, 36, 105-110. http://dx.doi.org/10.1016/j.medmal.2005.10.011

[14] Zimba, T.F., Nota, D.T., Langa, J.C., Leonel, C., Monteiro, G.S. and Coovadia, Y.M. (2009) The Aetiology of Acute Community Acquired Bacterial Meningitis in Children and Adults in Maputo, Mozambique. The Journal of Infection in Developing Countries, 3, 723-726. 